\title{
Spin analogues of proteins: scaling of 'folding' properties
}

\author{
Trinh Xuan Hoang, Nazar Sushko, Mai Suan Li and Marek Cieplak \\ Institute of Physics, Polish Academy of Sciences, Al Lotnikow 32/46, 02-668 Warsaw, Poland
}

Received 29 February 2000

\begin{abstract}
Reaching a ground state of a spin system is analogous to a protein evolving into its native state. We study the 'folding' times for various random Ising spin systems and determine characteristic temperatures that relate to the 'folding'. Under optimal kinetic conditions, the 'folding' timescale with the system size as a power law with a non-universal exponent. This is similar to what happens in model proteins. On the other hand, the scaling behaviour of the characteristic temperatures is different than in model proteins. Both in the spin systems and in proteins, the folding properties deteriorate with the system size.
\end{abstract}

\section{Introduction}

Recent numerical studies [1,2] indicate that characteristic folding times, $t_{\text {fold }}$, of model proteins grow with the number of amino acids, $N$, as a power law with an exponent which is nonuniversal-it depends on the class of sequences studied and on the temperature. The resulting deterioration of the folding properties also manifests itself in the way in which temperatures that relate to folding scale with $N$ [2]. There are two such characteristic temperatures: $T_{f}$ and $T_{\min }$. The first of these is a measure of the thermodynamic stability-it can be defined operationally as a temperature at which the probability of occupying the native (lowest energy) state passes $\frac{1}{2}$. The second temperature is one at which the folding kinetics is the fastest. At temperatures, $T$, below $T_{\min }$, glassy effects set in. Amino acid sequences that correspond to proteins should have a $T_{f}$ that is larger than $T_{\min }$, or at least comparable to $T_{\min }$. Otherwise the sequences are bad folders. Studies [2] of 2D and 3D lattice Go models [3] of proteins suggest that $T_{\min }$ grows with $N$ whereas $T_{f}$ first grows and then it either saturates or it grows at a lower rate than $T_{\min }$. There then exists a characteristic size, $N_{\mathrm{c}}$, at which $T_{\min }$ starts exceeding $T_{f}$ and for $N>N_{\mathrm{c}}$ the sequences necessarily become bad folders. This suggests the existence of a size-related limit to physiological functionality of proteins.

The question we ask in this paper is to what extent the scaling behaviour of $t_{\text {fold }}, T_{f}$, and $T_{\min }$ that was found in the lattice Go model of proteins is typical or, in other words, what are the classes of universality for these quantities. Specifically, we consider Ising spin systems: uniform ferromagnets (FM), disordered ferromagnets (DFM) and spin glasses (SG). DFM have recently been shown [4] to have a phase space structure, as described by the so-called disconnectivity graphs [5-7], quite akin to that characterizing proteins, at least for a small number of spins, $N$. SG, on the other hand, have been found to have the phase space structured as in random sequences of amino acids, which are bad folders. The spin systems do not 'fold' but an evolution into their ground states can be considered to be analogous to the folding process $[4,8]$ and $t_{\text {fold }}$ can be defined as the characteristic time needed to pass through the ground state for the first time, which generally does not coincide with a relaxation time. Thus 
$t_{\text {fold }}, T_{f}$, and $T_{\min }$ can be determined as for the proteins and we may additionally enquire how do $T_{f}$ and $T_{\min }$ relate to the effective critical temperature as determined from the specific heat and magnetic susceptibility.

Another motivation for considering the 'folding' in spin systems is that the analogies between spin systems and proteins have already permeated the language in which the physics of proteins is couched. It is not clear, however, to what extent these analogies are accurate when it comes to actual details. One qualitative concept, in this category, is that of the energy landscape $[9,10]$ : SG are said to have energy landscapes with many competing valleys but proteins should have landscapes which are funnel like. Another such concept is frustration [11]: the structural frustration in proteins should be 'minimal' whereas the frustration in the exchange couplings leads to the slow kinetics as found in SG. These concepts have been probed, e.g. in the random energy model [11] which again originated in the context of SG [12].

The basic message of this paper is that the spin-protein analogies are indeed valid but the details of the behaviour are usually distinct. What is analogous, for instance, is that the folding times have a characteristic U-shaped dependence on $T$ [13]. Furthermore, the folding properties are the best for small system sizes and then they deteriorate with $N$. In particular, the 'folding' times at $T_{\min }$ in spin systems do grow as a power law with $N$. On the other hand, both $T_{f}$ and $T_{\min }$ of simple spin systems generally decrease with $N$ and the nature of the phase transition is not a finite-size version of the first order as is the case with proteins.

The origins of the difference between spin systems and the Go models of proteins in the behaviour of $T_{f}$ and $T_{\min }$ remain to be elucidated. It should be noted that there are no kinematic constraints on flips of any spin whereas the possible moves in the protein folding process must preserve the chain connectivity and they have to depend on the actual conformation and thus on the history. The constrained character of the protein dynamics makes it acquire aspects of the packing problem, especially so if the native state is maximally compact—such as considered in the studies of scaling in model proteins. The packing aspects become insignificant when dealing with longer and longer $\alpha$-helices [14]. We illustrate this point here by considering a 2D lattice version of the $\alpha$-helices $(\mathrm{H})$ as described within the Go scheme and show that these objects indeed behave like spin systems when $N$ becomes larger. Note that the helices have the monomer-monomer interactions of a local kind. Thus the energy barrier against unfolding essentially does not depend on $N$, which is not expected of structures with more complex contacts.

Most of this paper, however, will be focused on systems described by the Ising spin Hamiltonian:

$$
\mathcal{H}=-\sum_{\langle i j\rangle} J_{i j} S_{i} S_{j}
$$

where $S_{i}= \pm 1$, and the exchange couplings, $J_{i j}$, connect nearest neighbours on the square and cubic lattices with the periodic boundary conditions. There are $L^{D}$ spins in the system where $D$ denotes the dimensionality and $L$ the linear size of the system. We consider four models of the exchange couplings: (1) SG in which the $J_{i j}$ are numbers drawn from the Gaussian probability distribution with a zero mean and a unit dispersion; (2) uniform FM with $J_{i j}=1$; (3) the DFM with $J_{i j}$ chosen as the absolute values of the Gaussian numbers; (4) the weakly disordered ferromagnets $\left(\mathrm{DFM}^{\prime}\right)$ with the $J_{i j}$ being random numbers between 0.9 and 1.1 . We find that it is the latter system which is the most protein-like.

In sections 2 and 3 we discuss the $T$ and $N$ dependences of the 'folding' times respectively. In section 4 we present results on the scaling behaviour of $T_{f}$ and $T_{\min }$ in systems SG, FM, DFM, DFM', and H. Finally, in section 5, we demonstrate that the temperatures we study are quite distinct from the critical temperature of the spin systems. 
a)

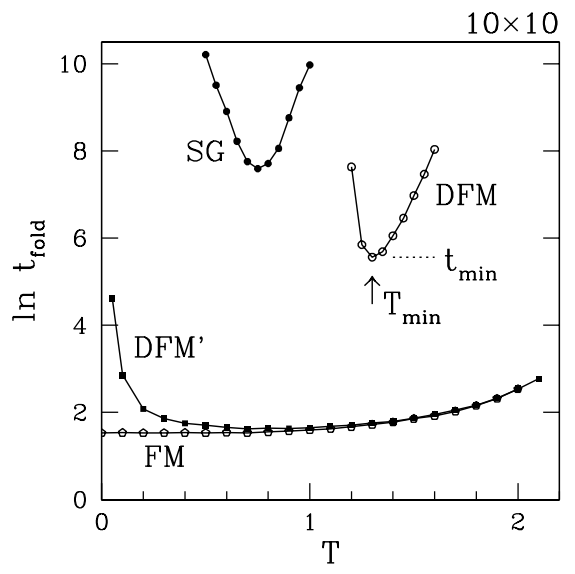

b)

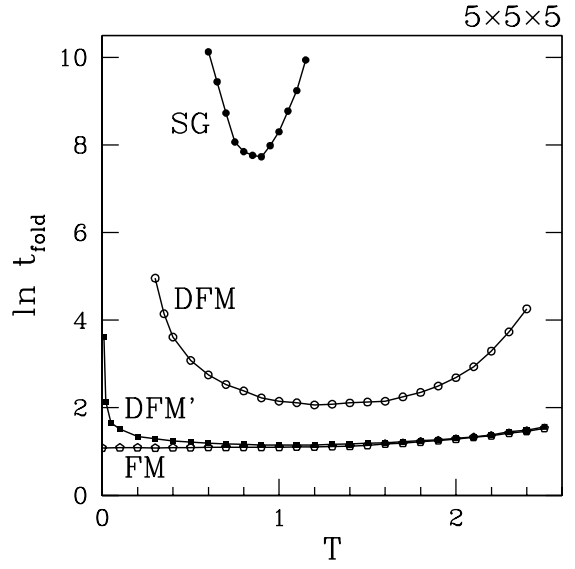

Figure 1. The temperature dependence of the characteristic 'folding' time for the (a) 2D and (b) 3D FM, DFM', DFM and SG Ising systems. Here, $L=10$ for the $2 \mathrm{D}$, and 5 for the $3 \mathrm{D}$ systems. The number of starting configurations is equal to 1000 .

\section{Temperature dependence of 'folding' times}

The concepts used in this paper are illustrated in figure 1 which shows the temperature dependence of the characteristic 'folding' time in the 2D and 3D Ising spin systems considered. In each category, data for a representative example system are shown. We obtain $t_{\text {fold }}$ by a standard Monte Carlo process in which one typically starts from 1000 random initial spin configurations and determines the median time to reach the ground state for the first time. The spins are updated sequentially and the 'folding' times are given in Monte Carlo steps per spin, i.e. the total number of spin updates divided by the number of spins. In the SG case, the ground state (or at least its close approximation) is obtained by multiple slow annealing processes followed by a quenching procedure. The general shape of the $T$ dependence is like in the protein systems - it corresponds to a U-shaped curve with a minimum at $T_{\min }$. The bigger the disorder, the higher the $T_{\min }$ - the DFM system has the highest $T_{\min }$ among the systems with the ferromagnetic ground state. Figure 1 also shows that the SG system has a lower value of $T_{\min }$ than the DFM. However, this does not reflect the degree of disorder since the two systems are different in nature. The fact that the SG system has a lower value of $T_{\min }$ than the corresponding DFM system is related to the fact that local energy barriers against spin flipping are generally higher in a DFM than in a SG due to a non-zero value of the average exchange coupling. The phase space structure of the uniform ferromagnet is so simple, containing few local energy minima, that the low temperature upturn does not develop down to $T=0$. In this 
case, we shall attribute a zero value to $T_{\min }$. A similar phenomenon has been observed in the lattice Go model with repulsive non-native contacts [15] for which only a few local minima are available. The shortest 'folding' time, $t_{\min }$, corresponds to $t_{\text {fold }}$ that is determined at $T_{\min }$.

The temperature dependence of the folding time on $N$ is also computed for the Go model of 'helices' on the 2D square lattice. A 'helical' native state for $N=16$ is shown at the top of figure 2. The meanders shown in the figure become longer and longer when $N$ increases. The Hamiltonian for the system is given by

$$
\mathcal{H}=\sum_{i<j} B_{i j} \Delta_{i j}
$$

where $\Delta_{i j}$ is either 1 or 0 , depending on whether the monomers $i$ and $j$ are nearest neighbours on the lattice but not nearest neighbours along the chain, or not. When $\Delta_{i j}$ is non-zero, the two monomers are said to form a contact. The definition of the Go model is that $B_{i j}$ is 1 for the native contacts (such as seen in figure 2) and 0 otherwise. Thus the properties of the system are determined entirely by the native conformation. The dynamics are defined in terms of a Monte Carlo process which satisfies the detailed balance conditions as explained in [2, 16, 17].

The lower part of figure 2 shows the characteristic U-shape dependence of $t_{\text {fold }}$ on $T$ for system $\mathrm{H}$. What is different compared to the models of maximally compact proteins is that the positions of both $T_{\min }$ and $T_{f}$ are seen to decrease with $N$-the point to which we shall come back in section 4 .

\section{Scaling properties of folding times at $T_{\min }$}

In order to study the scaling properties of disordered systems, such as the spin systems with random exchange couplings, one needs to consider ensembles of samples with properties which are similar statistically. Thus for each $N$ we have considered up to 50 samples and for each we have performed simulations of 'folding' in the Monte Carlo process. The median folding times as a function of $T$ have been calculated for each sample separately and we determine their fastest folding condition. Typically it is done by considering 1000 folding trajectories at each $T$. But for the FM and DFM' systems with a small size, up to 40000 trajectories at each $T$ have been used due to the broadness of the minimum. The value of $t_{\text {min }}$ has been determined at $T=T_{\min }$ that corresponded to a given sample and only then has the average of $t_{\min }$ over samples been calculated. Figure 3 shows the scaling of the average $t_{\min }$ for the 2D systems: FM, DFM', DFM, SG and H. Figure 4, on the other hand, deals with the 3D Ising systems.

All of the results are consistent with the power law:

$$
\left\langle t_{\min }\right\rangle \sim N^{\lambda}
$$

where the values of $\lambda$ are shown in table 1 . Interestingly, $\lambda$ for the spin systems depends much more strongly on the type of the spin system than on its dimensionality. On the other hand, in the Go models of proteins with the maximally compact native state, the dependence on dimensionality is strong: it is of order 6 and 3 in 2D and 3D respectively [1,2].

It should also be noted that for the $2 \mathrm{D}$ lattice 'helices', $\lambda \approx 4.59$ is substantially smaller than the exponent found for the Go proteins with the maximally compact native state which points to the role of the geometry. A recent study by Maritan et al [18] has indicated that the $\alpha$-helices fold the fastest among the objects with the same length and compactness. The presence of secondary motifs has also been shown to be responsible for differences in the folding behaviour between proteins and random globules [19]. Thus our result is in good agreement with those observations.

The strong dependence of $\lambda$ on the choice of the exchange couplings is similar to the lack of universality found in model proteins [1]. Also in analogy to the models of proteins, the 


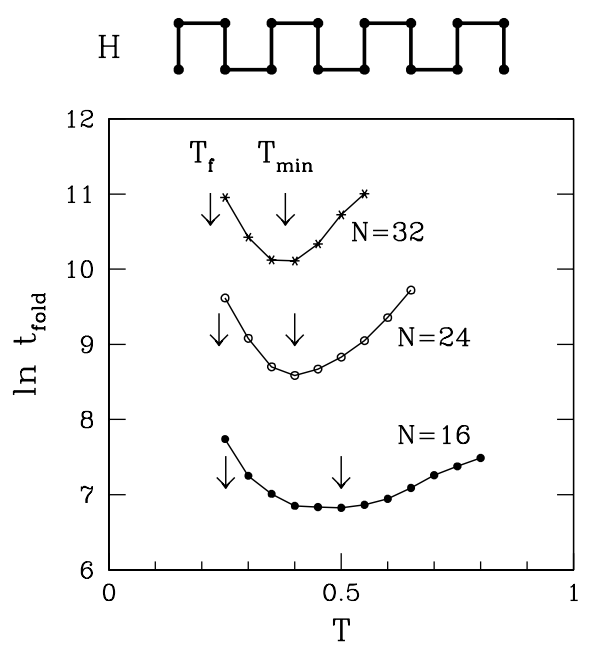

Figure 2. The top of the figure shows an example of a 'helical' conformation on the 2D lattice used in this paper. The conformation shown is for $N=16$. The lower part shows the dependence of $t_{\text {fold }}$ on $T$ for three indicated lengths of the 'helix'. The values of $T_{f}$ and $T_{\min }$ are marked by the arrows.

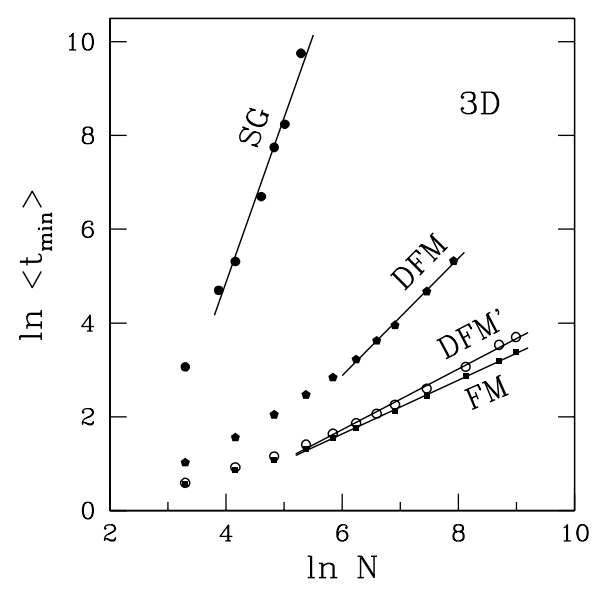

Figure 4. Scaling of $t_{\min }$ for $3 \mathrm{D}$ FM, DFM', DFM and SG.

scaling exponent depends on the temperature. Figure 5 shows that $t_{\text {fold }}$, evaluated not at $T_{\min }$ but at $T_{f}$, grows with an even bigger exponent or possibly the growth becomes exponential. This emphasizes the optimality of the kinetics at $T_{\min }$. In the DFM' case $t_{f}$ and $t_{\min }$ merge together because, as we shall see in the next section, the temperatures $T_{f}$ and $T_{\min }$ merge themselves.

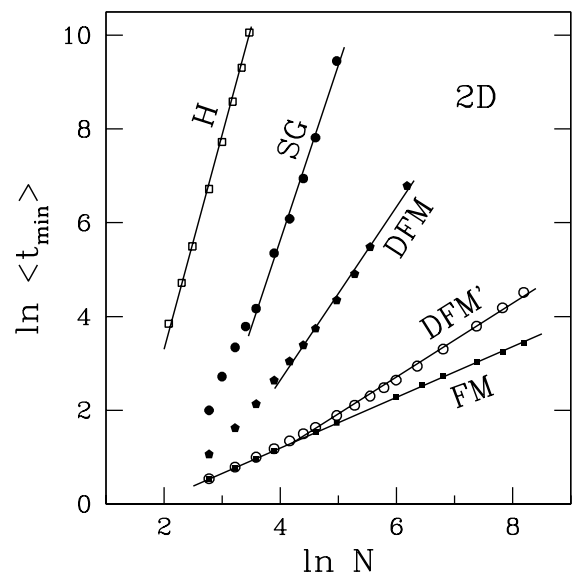

Figure 3. The scaling of $t_{\min }$ for 2D FM, DFM', DFM,

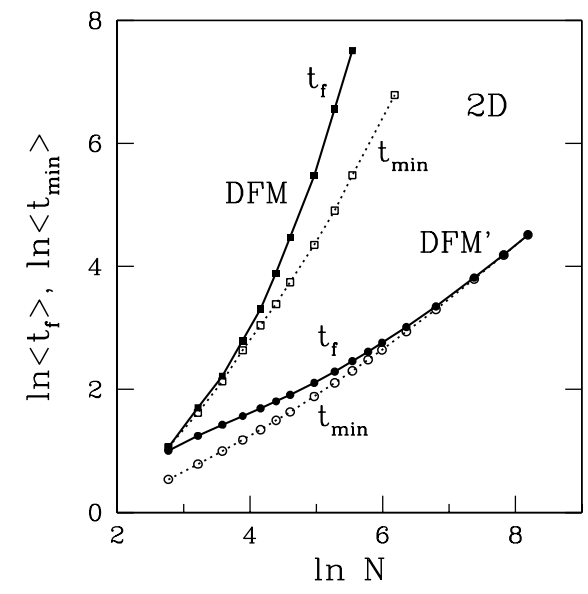

Figure 5. Scaling of $t_{f}$ for $2 \mathrm{D}$ DFM and $\mathrm{DFM}^{\prime}$ (solid curves) compared to the scaling of $t_{\min }$ (dotted curves). The effective slope for the last three data points corresponding to DFM is 3.54 . SG and the 'helices'. 
Table 1. The exponent $\lambda$ for the 2D and 3D spin systems and for the 2D Go models of helices. The symbol 'Go' in the table refers to the Go models with the maximally compact native states as studied in [2].

\begin{tabular}{lll}
\hline System & $\lambda(2 \mathrm{D})$ & $\lambda(3 \mathrm{D})$ \\
\hline FM & $0.54 \pm 0.01$ & $0.57 \pm 0.01$ \\
DFM $^{\prime}$ & $0.79 \pm 0.01$ & $0.65 \pm 0.02$ \\
DFM & $1.85 \pm 0.05$ & $1.25 \pm 0.05$ \\
SG & $3.73 \pm 0.15$ & $3.52 \pm 0.25$ \\
H & $4.59 \pm 0.08$ & - \\
Go & $6.3 \pm 0.2$ & $3.1 \pm 0.1$ \\
\hline
\end{tabular}

The possibility of a power law scaling for the folding time has been proposed theoretically by Thirumalai [21] (see also [22]) based on scaling concepts from polymer physics combined with some phenomenological assumptions. In particular, the power law scaling is argued to be relevant to proteins which fold through direct pathways with a nucleation mechanism. For indirect pathways, the folding time is determined primarily by activation processes with barriers which were argued to scale as $N^{1 / 2}$. There has also been a number of other studies of how a typical free-energy barrier, $B$, in model proteins scales with the number of monomers. All of these studies are phenomenological in nature and the barrier $B$ is often calculated at the folding transition temperature $T_{f}$. One assumes that the folding time is related to the barrier $B$ through an Arrhenius-like law: $\tau \sim \exp \left(B / k_{\mathrm{B}} T\right)$, as it is typically written for the relaxation time. In the random energy model [11], and also in another mean field approach for the Go model with a non-specific critical folding nucleus [20] the barrier scales linearly with $N$. Recently, Finkelstein and Badredtinov [23], and also Wolynes [24], have proposed a $N^{2 / 3}$ law by using a capillarity approximation. Gutin et al 's and our power laws for $t_{\text {fold }}$ obtained in simulations of the lattice proteins would formally correspond to a logarithmic dependence of the barrier on $N$ at the temperature of the fastest folding.

It should be noted, however, that the physics of folding coincides with that of equilibration only in the limit of low temperatures [16]. At high temperatures, for instance, the relaxation times are short but the folding times are long since the search for the ground state takes place primarily in the regions of phase space which are energetically remote from the target native state. Thus the behaviour of the barriers may have little bearing on the folding times at $T_{\min }$ which corresponds to the crossover between the physics of folding through equilibration and the physics of folding through a search for a state that takes place in equilibrium. At low temperatures, the roughness of the energy landscape becomes more and more significant, and the changed nature of the local barriers against the reconfiguration is expected to affect the scaling laws. Understanding the scaling behaviour of the folding time at $T_{\min }$ and at low temperatures still needs to be worked out—both in the protein and spin systems. The latter systems may prove to be easier conceptually and computationally.

\section{Scaling properties of $T_{f}$ and $T_{\min }$}

We now discuss the scaling of characteristic temperatures. $T_{\min }$ is determined from the kinetic data. $T_{f}$, on the other hand, is calculated by starting from the ground state and performing a long run that determines the equilibrium probability of the system staying in the ground state. The probabilities are determined as a function of $T$ and $T_{f}$ is obtained by an interpolation to where the value of $\frac{1}{2}$ is passed. For the spin systems, our results are based on up to 200 'unfolding' trajectories which last for up to 10000 Monte Carlo steps per spin. 


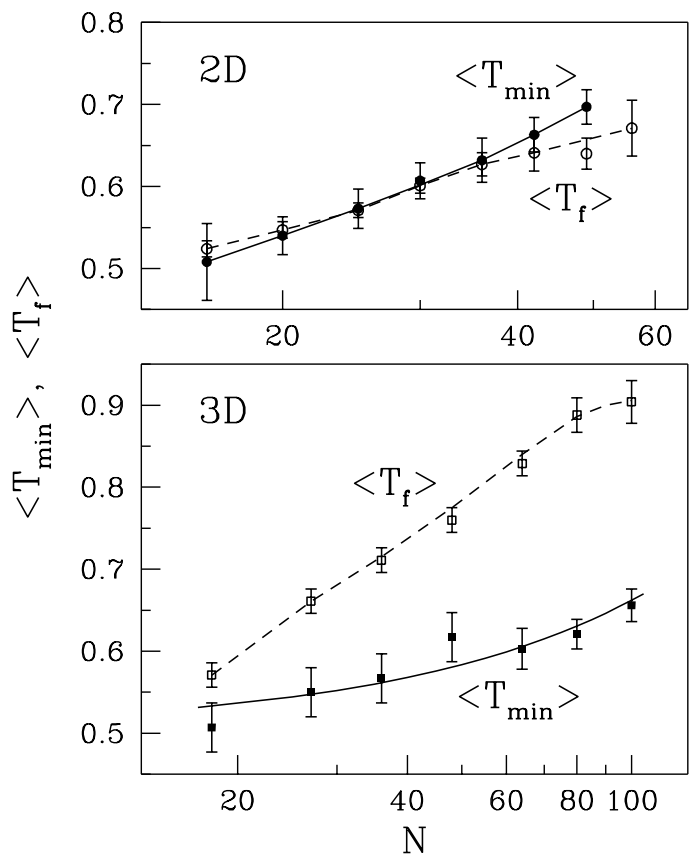

Figure 6. Scaling of $T_{f}$ and $T_{\min }$ for the $2 \mathrm{D}$ and 3D lattice Go models of proteins. Data points are taken from [2].

As a point of reference, we first consider the scaling properties of $T_{f}$ and $T_{\min }$ which were found in the 2D and 3D lattice Go models of proteins [2]. The corresponding data points are now shown, in figure 6 , as a function of $N$ on the logarithmic scale. Both $T_{f}$ and $T_{\min }$ grow with $N$. The data points suggest that $T_{\min }$ grows indefinitely - the larger the system size, the higher $T$ is needed to secure the optimal folding conditions. On the other hand, $T_{f}$ appears to tend to a saturation value- there is a limit to the thermodynamical stability. This finding is consistent with an analytical result obtained by Takada and Wolynes [20] for Go-like proteins studied within a droplet approximation.

Figure 7 shows the scaling of $T_{f}$ and $T_{\min }$ for the $2 \mathrm{D}$ lattice 'helix' system. At $N \leqslant 8$ the foldability is good but on increasing $N$, the behaviour is entirely different: the glassy effects decrease in importance $-T_{\min }$ decreases - but also the thermodynamic stability becomes more and more insignificant. The slopes for the $N$ dependence of the two temperatures are somewhat different and the corresponding plots may cross at some large value of $N$. Thus it is possible that good foldability can reappear at some large values of $N$-but at a very low $T$.

We now ask what kind of scaling behaviour of $T_{f}$ and $T_{\min }$ characterizes the spin systems? Figures 8-11, for systems FM, DFM', DFM and SG respectively, demonstrate that in no case is the scaling like that for the Go lattice models with the maximally compact native state but in some cases it is akin to the behaviour exhibited by the 2D 'helix'.

Both for the 'helix' and for all of the spin systems studied here, $T_{f}$ decreases with $N$ monotonically which is not what happens in the Go models of proteins. This difference in behaviour can be traced to the following observation. $T_{f}$ is defined through the equation

$$
P_{N}=\frac{1}{1+\sum_{l}^{\prime} \exp \left(-\left(E_{l}-E_{N}\right) / k_{\mathrm{B}} T_{f}\right)}=\frac{1}{2}
$$

where $P_{N}$ is a probability of being in the ground state, $E_{N}$ is the energy of the ground state, $E_{l}$ is the energy of an $l$ th state, and the sum written in the denominator excludes the ground state. 


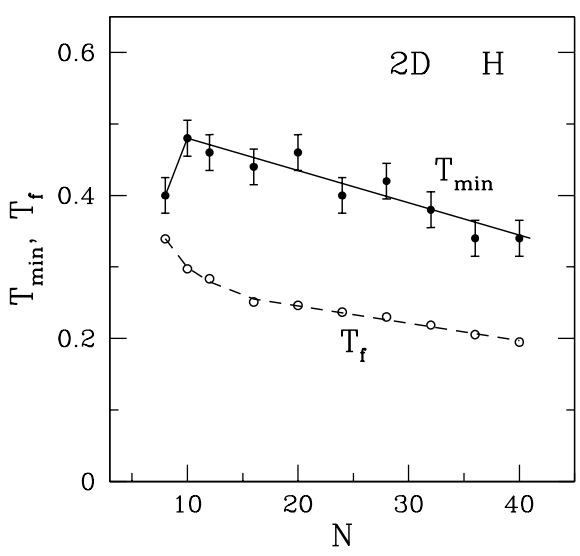

Figure 7. The same as in figure 6 but for 2D Go models of the 'helices'.
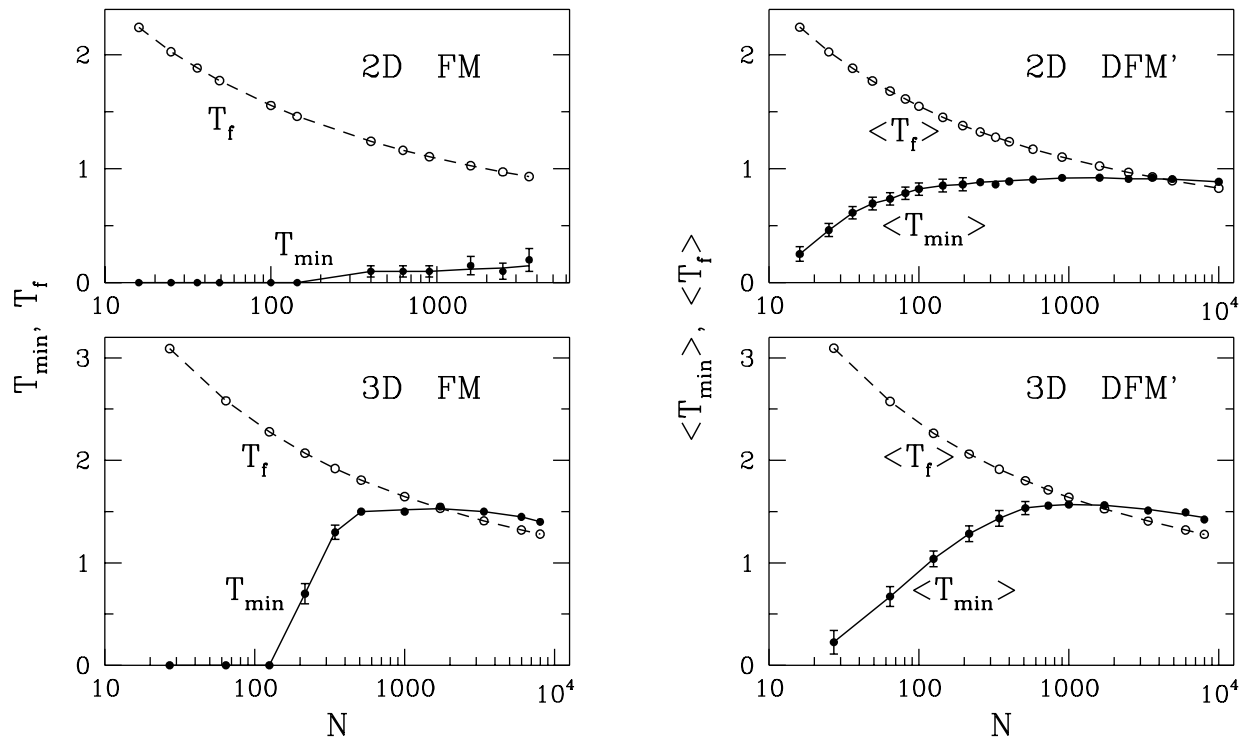

Figure 8. The dependence of $T_{f}$ and $T_{\min }$ of 2D and 3D FM on $N$.

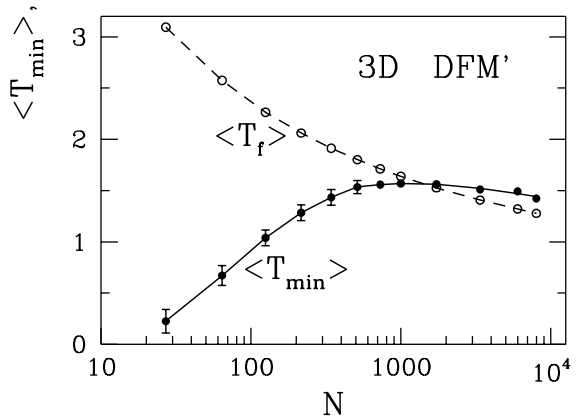

Figure 9. The same as in figure 8 but for the $\mathrm{DFM}^{\prime}$ systems.

At temperatures which do not exceed $T_{f}$, the sum is dominated by the low-energy excitations. In the spin systems and in the 'helix', energies of these excitations do not depend on $N$. For instance, in the Ising case they are of order $2 z J$, where $z$ is the coordination number and $J$ denotes a characteristic value of the exchange interactions. It is only the number of terms in the sum itself that grows with $N$. This leads to $T_{f}$ decreasing with $N$. On the other hand, in the model proteins, the energies of the excitations typically do depend on $N$ which may have a competing effect on $T_{f}$ relative to the impact of the number of states.

We now turn to discussion of the scaling properties of $T_{\min }$. From figures $8-11$ it is clear that it has opposite tendencies for proteins and spin systems. For the 2D FM and DFM' systems one observes an increase followed by saturation. In all other spin systems, instead of 

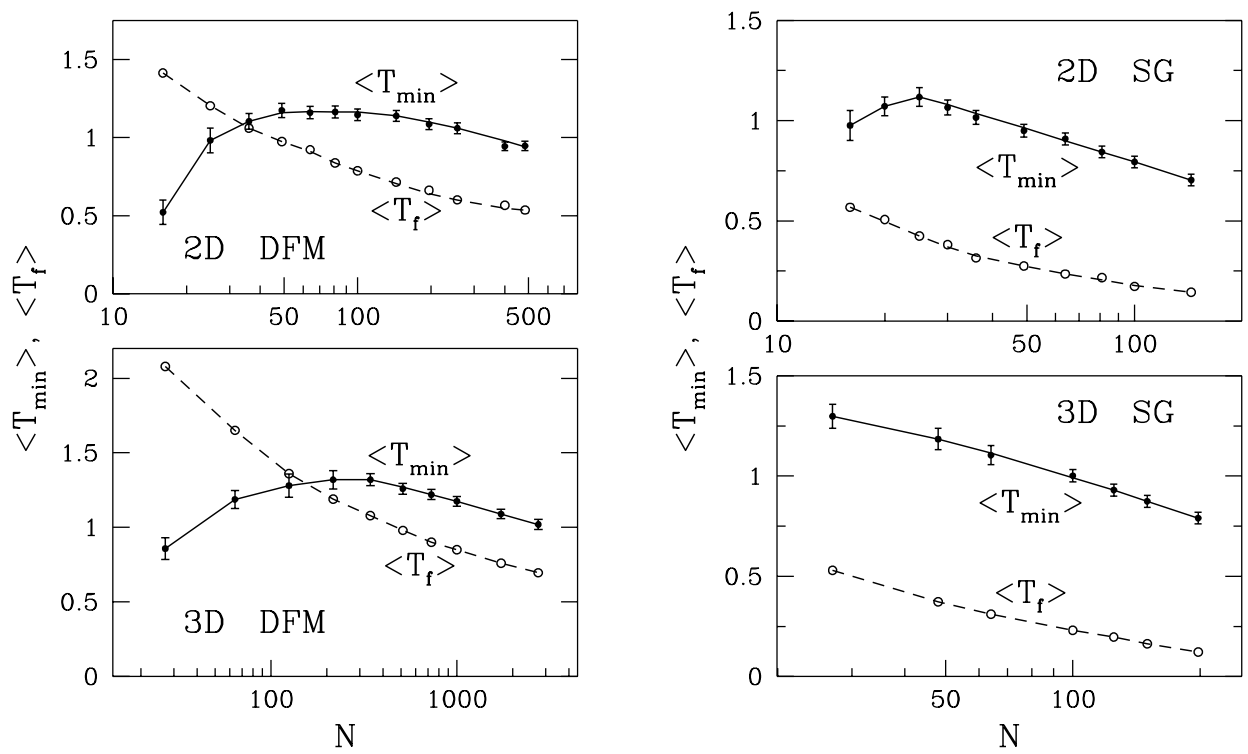

Figure 10. The same as in figure 8 but for the DFM

Figure 11. The same as in figure 8 but for the SG systems. systems.

the saturation, one observes a maximum followed by an asymptotic decrease. The difference may reflect the presence of the kinematic constraints on possible moves in proteins due to their polymeric nature. Such constraints may cause the emergence of barriers which depend on $N$ and result in a growing $T_{\min }$. In spin systems such kinematic constraints do not exist: each spin configuration has $N$ possible ways to move out with a cost which does not depend on $N$. Such a high number of degrees of freedom gives the spin systems a large flexibility to cross from local minima to local minima. Thus there is no potential for an indefinite growth of $T_{\min }$. The initial growth, in the random systems, reflects on the role of the growing number of local energy minima which may form kinetic traps and make the kinetics glassy. Yet the asymptotic decrease of $T_{\min }$ suggests that the relevant traps do not need a $N$-dependent energy to overcome or points to some entropic effect.

The case of the 'helix' system may appear puzzling at first glance since it possesses polymeric constraints and yet they do not lead to a growing $T_{\min }$. Note that, in contrast to the model proteins with maximally compact native states [2], the Hamiltonian for the 'helix' contains terms related only to the local contacts. Thus the chain is much more flexible when it is not tightly packed in its native state. The energy barriers against escaping from the traps do not depend on the chain length, and therefore the 'helix' exhibits spin-like properties. High energetic barriers in proteins are often associated with breaking of some tertiary contacts.

\section{The specific heat and susceptibility}

We now compare $T_{f}$ and $T_{\min }$ with the usual critical temperatures that characterize spin systems. We focus on the properties of the specific heat, $C$, and susceptibility, $\chi$, defined through

$$
C=\frac{\left\langle E^{2}\right\rangle-\langle E\rangle^{2}}{N T^{2}}
$$




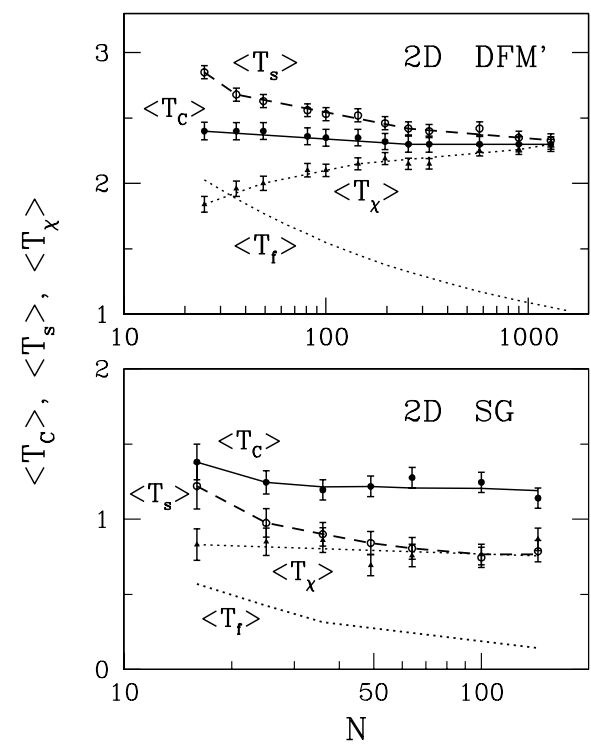

Figure 12. The size dependence of $T_{C}, T_{\chi}, T_{s}$ and $T_{f}$ for the $2 \mathrm{D} \mathrm{DFM}^{\prime}$ and $\mathrm{SG}$ systems.

and

$$
\chi=\frac{\left\langle M^{2}\right\rangle-\langle M\rangle^{2}}{N T}
$$

respectively, where $M$ is the magnetization. The temperatures at which $C$ and $\chi$ have a maximum will be denoted as $T_{C}$ and $T_{\chi}$, respectively.

In addition, and in analogy to the proteins [25], we study the structural fluctuations

$$
\Delta \chi_{s}=\left\langle\chi_{s}^{2}\right\rangle-\left\langle\chi_{s}\right\rangle^{2}
$$

which are defined in terms of the structural overlap function

$$
\chi_{s}=\frac{1}{N}\left|\sum_{i=1}^{N} S_{i} S_{i}^{(N)}\right|
$$

where $\left\{S_{i}^{(N)}\right\}$ is the spin configuration in the ground state. (For the FM $\chi_{s}$ is the same as the absolute value of the magnetization per spin.) These fluctuations also have a maximum at some temperature which will be denoted by $T_{s}$. It has been suggested [25] that for proteins $T_{s}$ should be about $T_{f}$ and a small difference between $T_{s}$ and $T_{C}$ is a signature of fast folding.

All of these thermodynamic quantities are averaged over 10 to 20 samples and 100 trajectories for each. In each trajectory, the first 5000 to 10000 Monte Carlo steps per spin are spent for equilibration. The trajectories were then further evolved between 20000 and 50000 steps per spin. The lower values above refer to the $\mathrm{DFM}^{\prime}$ system, and the higher to the SG system.

Figure 12 shows the scaling behaviour of $T_{C}, T_{\chi}$, and $T_{s}$ for the $2 \mathrm{D}$ DFM' and SG systems. In the case of $\mathrm{DFM}^{\prime}$, the three temperatures converge to one common critical temperature. Note that none of these temperatures has anything to do with $T_{f}$ or $T_{\min }$. In the SG system, $T_{s}$ and $T_{\chi}$ tend to separate asymptotics than $T_{C}$ but again none of these temperatures coincides with $T_{f}$ or $T_{\min }$. The physics of folding is not related to the critical phenomena. It should be noted that a phase transition in SG shows as a singularity in the nonlinear susceptibility. In the 2D SG system, the peak position in the nonlinear susceptibility should be at $T=0$ for any system size [26]. 
In summary, we have studied Ising spin systems from the perspective of protein folding. We have demonstrated that there exist many similarities between the spin and polymeric systems. In particular, we have shown that both kind of systems have the property of a power law scaling of the folding time at $T_{\min }$ as a function of $N$. We point out that this holds, independent of whether the system is a good or bad folder and is thus some universal feature of folding.

Among the random spin systems studied here, the DFM' systems have the biggest range of the small $N$ values at which $T_{f}$ is larger than $T_{\min }$, both in $2 \mathrm{D}$ and $3 \mathrm{D}$. Thus these smallsized systems are the best analogues of good folders and can serve as models that mimic the physics of proteins. SG of any size, on the other hand, do indeed mimic the physics of random heteropolymers. Asymptotically, though, each random spin system is a bad 'folder'.

\section{Acknowledgments}

This work was supported by KBN (Grant No 2P03B-025-13). MSL thanks H Rieger for useful discussions.

\section{References}

[1] Gutin A M, Abkevich V I and Shakhnovich E I 1996 Phys. Rev. Lett. 775433

[2] Cieplak M, Hoang T X and Li M S 1999 Phys. Rev. Lett. 831684

[3] Go N and Abe H 1981 Biopolymers 201013

[4] Garstecki P, Hoang T X and Cieplak M 1999 Phys. Rev. E 603219

[5] Becker O M and Karplus M 1997 J. Chem. Phys. 1061495

[6] Wales D J, Miller M A and Walsh T R 1998 Nature 394758

[7] Miller M A and Wales D J 1999 J. Chem. Phys. 1116610

[8] Frauenfelder H and Wolynes P G 1994 Phys. Today 4758

[9] Bryngelson J D, Onuchic J N, Socci N D and Wolynes P G 1995 Proteins 21167

[10] Onuchic J N, Luthey-Schulten Z and Wolynes P G 1997 Ann. Rev. Phys. Chem. 48545

[11] Bryngelson J D and Wolynes P G 1987 Proc. Natl Acad. Sci. USA 847524

[12] Derrida B 1980 Phys. Rev. Lett. 4579

[13] Socci N D and Onuchic J N 1994 J. Chem. Phys. 1011519 see also Cieplak M and Banavar J R 1997 Fold. Des. 2235

[14] Hoang T X and Cieplak M 2000 J. Chem. Phys. 156851

[15] Li M S and Cieplak M 2000 Eur. Phys. J. B 14787

[16] Cieplak M, Henkel M, Karbowski J and Banavar J R 1998 Phys. Rev. Lett. 803654

[17] Cieplak M, Maritan A and Banavar J R 1999 J. Cond. Mat. 2369

[18] Maritan A, Micheletti C and Banavar J R 2000 Phys. Rev. Lett. 843009

[19] Micheletti C, Banavar J R, Maritan A and Seno F 1999 Phys. Rev. Lett. 823372

[20] Takada S and Wolynes P G 1997 J. Chem. Phys. 1079585

[21] Thirumalai D 1995 J. Physique 51457

[22] Camacho C J and Thirumalai D 1996 Europhys. Lett. 35627

[23] Finkelstein A V and Badredtinov A Y 1997 Fold. Des. 2115

[24] Wolynes P G 1997 Proc. Natl Acad. Sci. USA 946170

[25] Klimov D K and Thirumalai D 1996 Phys. Rev. Lett. 764070

Camacho C J and Thirumalai D 1993 Proc. Natl Acad. Sci. USA 906369

[26] Rieger H, Santen L, Blasum U, Diehl M, Junger M and Rinaldi G 1996 J. Phys. A: Math. Gen. 293939 